\title{
Risk of colonic diverticular rebleeding according to endoscopic appearance
}

\section{(ㄷ)(잉}

\author{
Authors \\ Mikitaka Iguchi' ${ }^{1}$ Masayuki Kitano ${ }^{1}$ \\ Institutions \\ 1 Second Department of Internal Medicine, Wakayama \\ Medical University, Wakayama, Japan \\ 2 Department of Gastroenterology, Wakayama Rosai \\ Hospital, Wakayama, Japan
}

Koki Kawanishi ${ }^{1}$, Jun Kato ${ }^{1}$, Tetsuhiro Kakimoto², Takeshi Hara², Takeichi Yoshida', Yoshiyuki Ida', Takao Maekita ${ }^{1}$,

submitted 24.8.2017

accepted after revision 20.10.2017

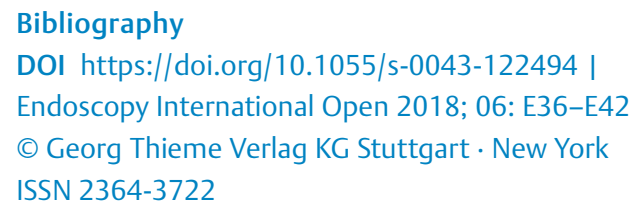

Corresponding author Jun Kato, Second Department of Internal Medicine, Wakayama Medical University, 811 Kimiidera, Wakayama City, Wakayama 641-0012, Japan

Fax: +81-73-445-3616

katojun@wakayama-med.ac.jp

\section{ABSTRACT}

Background and study aims Re-commencement of bleeding (rebleeding) of colonic diverticula after endoscopic hemostasis is a clinical problem. This study aimed to examine whether endoscopic visibility of colonic diverticular bleeding affects the risk of rebleeding after endoscopic hemostasis.
Patients and methods We performed a retrospective review of endoscopic images and medical charts of patients with colonic diverticular bleeding who underwent endoscopic hemostasis. Endoscopic visibility was classified into two types according to visibility of the source of bleeding; source invisibility due to bleeding or attached hematin (type 1), or endoscopically visible responsive vessels (type 2 ). Rebleeding rates within one year after initial hemostasis were examined.

Results of 93 patients with successful endoscopic hemostasis, 38 (41\%) showed type 1 visibility, while the remaining presented type 2 . All patients received hemostasis with clipping, rebleeding developed in 20 patients (22\%). Type 1 visibility was more likely to be observed in patients with rebleeding ( $65 \%$ vs. $34 \%, P=0.013)$. Multivariate analysis revealed that after endoscopic hemostasis, type 1 visibility (invisible source) was the only independent risk factor for colonic diverticular rebleeding (odds ratio, 3.05; $95 \%$ confidence interval, 1.03-9.59, $P=0.044$ ). Kaplan-Meier curve showed the cumulative incidence of rebleeding was significantly higher in patients with type 1 visibility than those with type 2 visibility ( $P=0.0033$, log-rank test)

Conclusion Hemostasis by clipping for colonic diverticular bleeding without definite observation of the source of bleeding may not be sufficiently effective. Other hemostatic methods, including band ligation, should be considered when the source of bleeding is unclear.

\section{Introduction}

In developed countries including Japan and the United States, the incidence of colonic diverticular bleeding is increasing along with the increase in the aging population, because diverticula are more likely to occur in older subjects. In addition, older patients often have comorbidities that contribute to susceptibility to bleeding including hypertension, arteriosclerosis and regular use of anti-thrombotic agents or nonsteroidal antiinflammatory drugs (NSAIDs) [1 - 3].
Colonic diverticular bleeding is the most common cause of lower gastrointestinal bleeding [4], and it has been reported that between $70 \%$ and $90 \%$ of patients with colonic diverticular bleeding show spontaneous arrest of bleeding [5]. However, the remaining cases are likely to present severe or continuous bleeding and require hemostatic procedures including endoscopic treatment, surgery, or trans-arterial embolization [6].

Rebleeding from diverticula after successful endoscopic hemostasis is also a critical problem and the incidence was reported to be between $10.8 \%$ and $43.4 \%$ [6, 7]. Risk factors for colo- 

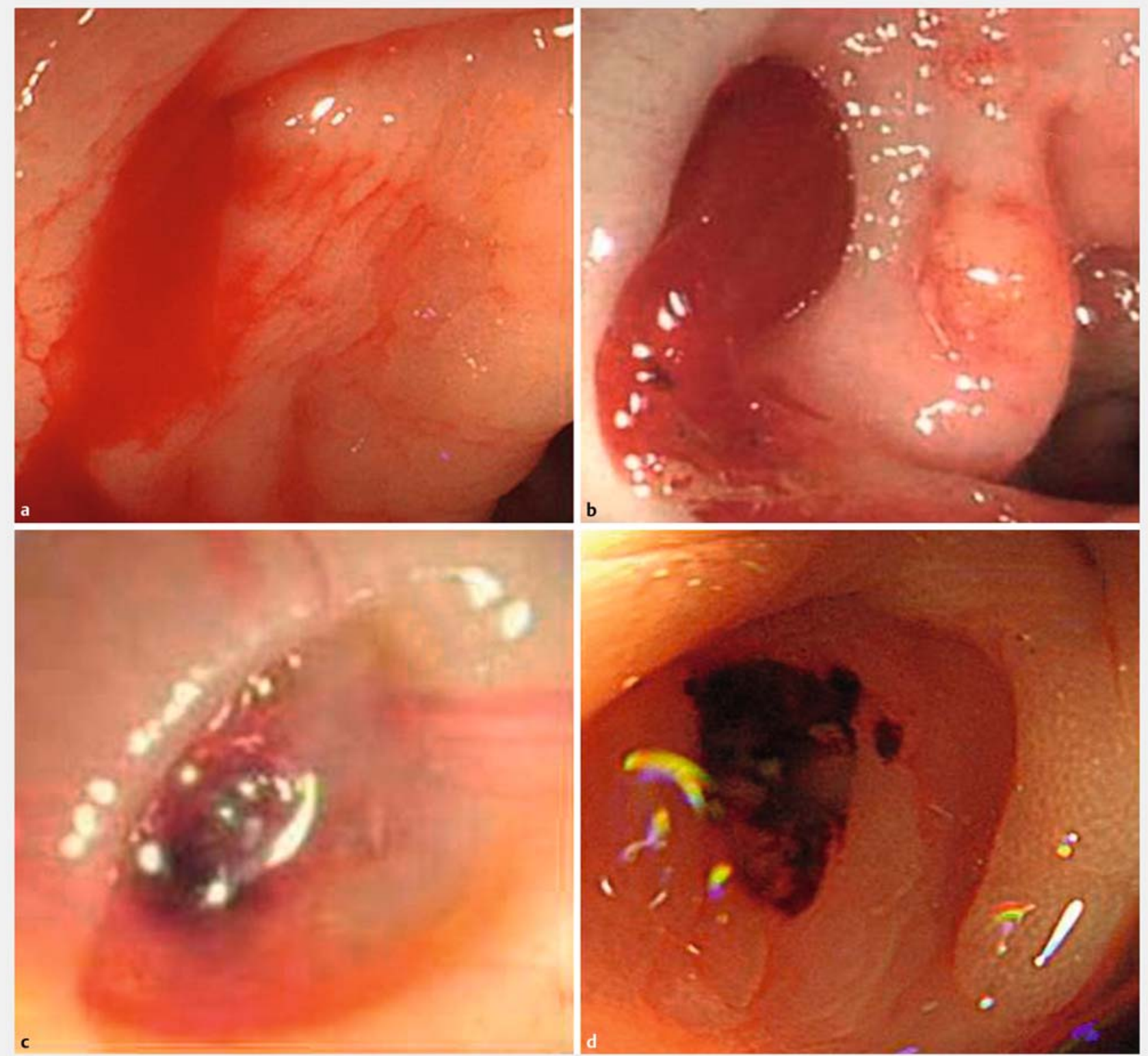

Fig. 1 a Active bleeding from the diverticulum. b Massive hematin attached to the base. $\mathbf{c}$ Visible blood vessel in the diverticulum base. d Black spot in the diverticulum base.

nic diverticular rebleeding have been identified: bleeding from the right colon, shock, hypertension, hyperlipidemia, and the use of NSAIDs or anti-thrombotic drugs [7-10]. However, endoscopic visibility of diverticula with bleeding has not been evaluated in association with rebleeding risk, although variety is observed in the appearance of diverticular bleeding, including active spurting from diverticula and vessel exposure in the diverticular bed.

The current study aimed to assess whether the difference in endoscopic visibility of diverticula with bleeding affects the risk of rebleeding after successful endoscopic hemostasis.

\section{Patients and methods}

\section{Patients and study design}

This is a retrospective analysis of patients who underwent endoscopic hemostasis for diverticular bleeding at Wakayama Rosai Hospital, a secondary emergency hospital in Japan, between June 2005 and May 2017. For colonoscopy and hemostatic procedures, the latest guidelines such as the ACG guidelines [11] were followed. Adequate colonic cleansing was rendered to all cases except when the patient condition aggravated due to massive hemorrhage.

We included patients who underwent successful endoscopic hemostasis for diverticular bleeding with definite endoscopic 

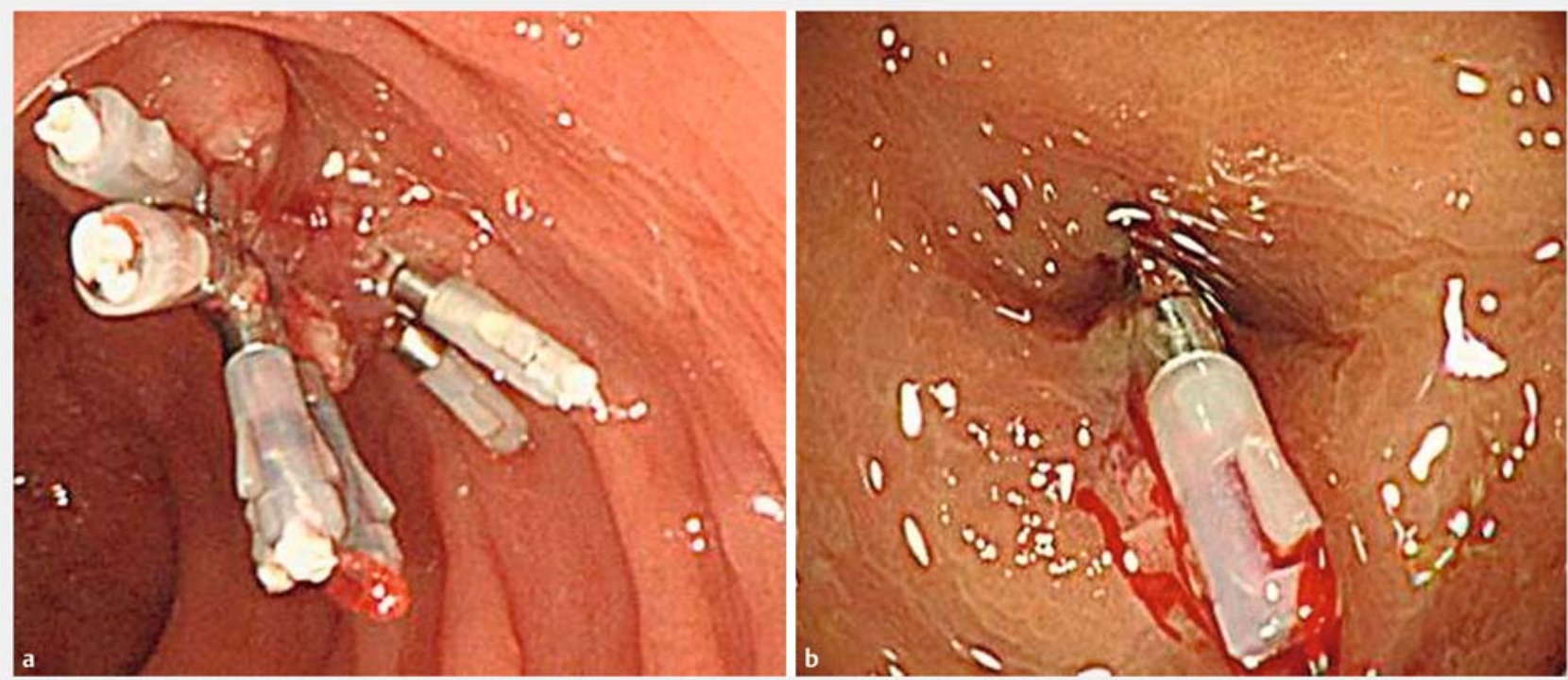

> Fig. 2 a "Reefing method" for diverticular bleeding invisible bleeding source, b "Direct clip placement" for diverticular bleeding with definite detection of bleeding source.

detection of a responsible locus. Patients who received endoscopic hemostatic procedures without definite detection of a bleeding site, such as clipping for multiple diverticula suspicious of bleeding, were not included. We also excluded those with any further hemostatic procedure, including interventional radiology or surgery. Another exclusion was less than one year of follow-up without rebleeding.

From medical charts we collected information about demographics, endoscopic visibility of bleeding, location of bleeding (right-side including the cecum, ascending, and transverse colon vs. left-side), blood transfusion, time between admission and endoscopy, comorbidities including hypertension, hyperlipidemia, diabetes mellitus, heart diseases, liver cirrhosis, impaired renal function (estimated glomerular filtration rate $<30 \mathrm{~mL} / \mathrm{min}$ ), pulmonary diseases and stroke, medication, and endoscopist's experience. The evaluated outcomes were the rebleeding rate within 1 year after initial hemostasis and the time to rebleeding. Patients with rebleeding were divided into two groups: early rebleeding group (within 30 days) and late rebleeding group (more than 30 days and within one year), and factors associated with rebleeding and early rebleeding were analyzed.

The Ethics Committee of Wakayama Rosai Hospital approved this retrospective study, which conformed to the provisions of the Declaration of Helsinki (as revised in Fortaleza, Brazil, October 2013).

\section{Endoscopic visibility of diverticular bleeding and hemostasis}

During all endoscopic procedures, still images were stored digitally, and those images were evaluated retrospectively for analysis by two of the authors (K.K. and J.K.). We classified endo- scopic visibility of diverticular bleeding into two groups according to visibility of bleeding source.

In type 1 , active bleeding, the bleeding source could not be seen directly due to spurting bleeding from a diverticulum ( $\triangleright$ Fig. 1a) or massive hematin attached to the base ( $\triangleright$ Fig. 1b).

In type 2, non-active bleeding, we could directly observe accurate bleeding source including visible blood vessels ( $>$ Fig.1c) or a black spot in the diverticulum base ( $\mathbf{F i g . 1 d}$ ).

Endoscopic hemostasis was performed by hemoclips for both types of bleeding. In cases of the type 1, the bleeding diverticulum was closed by hemoclips ( $\mathbf{F i g . 2 a}$ ) due to invisibility of bleeding source, and this procedure was reported as either "reefing method" or "indirect clip placement" [12]. In contrast, in cases of type 2 endoscopic visibility, hemoclips were directly applied to the exact point of bleeding (the direct clip placement [12]) ( Fig. 2b). For the exact hemoclip placement, the super short clips, HX-610-135XS (Olympus, Tokyo, Japan), were used. The clips fit for the narrow space owing to the short arm length ( $4 \mathrm{~mm})$.

\section{Definition of rebleeding}

Rebleeding was confirmed directly by colonoscopy, and only bleeding from the same diverticulum that previously received endoscopic hemostasis was defined as rebleeding. The consistency of the diverticula between previous bleeding and rebleeding was evaluated with endoscopic reports and stored endoscopic images. The information including the distance from the anus and meticulous comparisons of stored images enabled to confirm that the diverticulum with the initial bleeding was identical to that with rebleeding, even in cases that the stigmata of recent bleeding had already disappeared. Cases where the bleeding occurred at diverticula other than where in- 
- Table 1 Baseline characteristics of patients with colonic diverticular bleeding who underwent endoscopic hemostasis.

\begin{tabular}{|c|c|}
\hline \multicolumn{2}{|l|}{ Patients } \\
\hline Total & 93 \\
\hline Age (years) & $66(35-94)$ \\
\hline \multicolumn{2}{|l|}{ Gender } \\
\hline - Male & $72(77 \%)$ \\
\hline - Female & $21(23 \%)$ \\
\hline \multicolumn{2}{|l|}{ Classification of bleeding } \\
\hline - Type 1 & $38(41 \%)$ \\
\hline - Type 2 & $55(59 \%)$ \\
\hline \multicolumn{2}{|l|}{ Location of bleeding } \\
\hline - Right side of the colon & $69(74 \%)$ \\
\hline - Left side of the colon & $24(26 \%)$ \\
\hline Blood transfusion & $48(52 \%)$ \\
\hline \multicolumn{2}{|l|}{ Time to endoscopy } \\
\hline - $<12$ hours & $53(57 \%)$ \\
\hline - $\geq 12$ hours & $40(43 \%)$ \\
\hline \multicolumn{2}{|l|}{ Concomitant diseases } \\
\hline - Hypertension & $51(55 \%)$ \\
\hline - Hyperlipidemia & $48(52 \%)$ \\
\hline - Diabetes mellitus & $27(29 \%)$ \\
\hline - Heart diseases & $19(20 \%)$ \\
\hline - Liver cirrhosis & $14(15 \%)$ \\
\hline - Impaired renal function & $14(15 \%)$ \\
\hline - Pulmonary diseases & $8(9 \%)$ \\
\hline - Stroke & $6(6 \%)$ \\
\hline \multicolumn{2}{|l|}{ Medication } \\
\hline - Anti-thrombotic agents & $25(27 \%)$ \\
\hline - Non-steroidal anti-inflammatory drugs & $23(25 \%)$ \\
\hline - Proton pump inhibitor & $18(19 \%)$ \\
\hline \multicolumn{2}{|l|}{ Endoscopist's experience } \\
\hline - $\geq 3000$ colonoscopies & $53(57 \%)$ \\
\hline - <3000 colonoscopies & $40(43 \%)$ \\
\hline
\end{tabular}

itial bleeding was seen were not regarded as rebleeding in this study, and were included in the not-rebleeding group.

\section{Statistical analysis}

The primary outcome was rebleeding within one year after hemostasis. Differences between patients with and without colonic diverticular rebleeding after endoscopic hemostasis were determined using the chi-square test and Mann-Whitney $U$ test as appropriate. Using multivariate logistic regression anal- ysis, independent risk factors for the development of colonic diverticular rebleeding after endoscopic hemostasis were identified, and parameters with $P<0.05$ in univariate analysis were included. Cumulative rebleeding rates after hemostasis were calculated using the Kaplan-Meier method and evaluated by the log-rank test.

A $P$ value $<0.05$ was considered statistically significant, and odds ratio (OR) with $95 \%$ confidence intervals (Cls) were determined. Data were statistically analyzed using JMP version 9 software (SAS Institute, Cary, NC, USA).

\section{Results}

During the study period, 93 patients with diverticular bleeding underwent successful initial endoscopic hemostasis, and were followed-up for at least one year or until rebleeding. > Table 1 shows the clinical characteristics of analyzed patients (male, 72 (77\%); median age 66 years, range 35-94 years). As for type of endoscopic visibility, $38(41 \%)$ patients were classified as type 1 , while the remaining presented as type 2 . The location of bleeding was the right-side colon in 69 (74\%) patients. More than half of patients had comorbidities including hypertension (55\%) and impaired renal function (15\%). Anti-thrombotic agents and NSAIDs were administered to 25 (27\%) and 23 (25\%), respectively. An experienced doctor ( $\geq 3000$ colonoscopies) executed hemostasis in more than half of the cases. Rebleeding was observed in 20 (22\%) patients within 1 year after endoscopic hemostasis: 8 early rebleeding and 12 late rebleeding. In the early rebleeding group, type 1 was more frequently observed (6 vs. 2, $P=0.040$ ), while in the late bleeding group, statistical difference in frequency was not observed between type 1 and type 2 ( 7 vs. 5, $P=0.19$ ).

- Table 2 compares the clinical characteristics between patients with and without rebleeding. Type 1 visibility was more likely to be observed in patients with rebleeding (65\% vs. $34 \%$, $P=0.013)$. Impaired renal function and anti-thrombotic agent administration were also frequently observed in patients with rebleeding. There were no statistical differences in clinical characteristics and colonic cleansing state between type 1 and type 2 groups.

Multivariate analysis revealed that type 1 visibility was the only independent risk factor for both colonic diverticular rebleeding within one year and early rebleeding (within 30 days) after endoscopic hemostasis (OR, 3.05; $95 \% \mathrm{Cl}, 1.03-9.59, P=$ 0.044 , and $\mathrm{OR}, 6.37 ; 95 \% \mathrm{Cl}, 1.26-32.59, P=0.022$, respectively) ( $\triangleright$ Table 3 and $>$ Table 4 ). Kaplan-Meier curve showed that the cumulative incidence of rebleeding was significantly higher in patients with type 1 visibility than in patients with type 2 visibility ( $P=0.0033$, log-rank test) ( $>$ Fig. 3 ). These results suggest that endoscopic visibility of diverticular bleeding significantly correlates with susceptibility to rebleeding, and that impossibility to directly observe the bleeding source is a risk of rebleeding. 
- Table 2 Differences between patients with and without rebleeding.

\begin{tabular}{|c|c|c|c|}
\hline & \multicolumn{3}{|l|}{ Rebleeding } \\
\hline & Yes $(n=20)$ & No $(n=73)$ & $P$ \\
\hline Age (years) & $67(39-88)$ & $65(35-94)$ & 0.84 \\
\hline \multicolumn{4}{|l|}{ Gender } \\
\hline - Male & $15(75 \%)$ & $57(78 \%)$ & 0.77 \\
\hline \multicolumn{4}{|l|}{ Appearance of bleeding } \\
\hline - Type 1 & $13(65 \%)$ & $25(34 \%)$ & $0.013^{1}$ \\
\hline \multicolumn{4}{|l|}{ Location of bleeding } \\
\hline - Right side of the colon & $14(70 \%)$ & $55(75 \%)$ & 0.63 \\
\hline Blood transfusion & $13(65 \%)$ & $35(48 \%)$ & 0.18 \\
\hline \multicolumn{4}{|l|}{ Time to endoscopy } \\
\hline - $\leq 12$ hours & $12(60 \%)$ & $41(56 \%)$ & 0.76 \\
\hline \multicolumn{4}{|l|}{ Concomitant diseases } \\
\hline - Hypertension & $14(70 \%)$ & $37(51 \%)$ & 0.12 \\
\hline - Hyperlipidemia & $12(60 \%)$ & $36(49 \%)$ & 0.4 \\
\hline - Diabetes mellitus & $7(35 \%)$ & $20(27 \%)$ & 0.51 \\
\hline - Heart diseases & $3(15 \%)$ & $16(22 \%)$ & 0.5 \\
\hline - Liver cirrhosis & $3(15 \%)$ & $11(15 \%)$ & 0.99 \\
\hline - Impaired renal function & $7(35 \%)$ & $7(10 \%)$ & $0.0049^{1}$ \\
\hline - Pulmonary diseases & $2(10 \%)$ & $6(8 \%)$ & 0.8 \\
\hline - Stroke & $1(5 \%)$ & $5(7 \%)$ & 0.77 \\
\hline \multicolumn{4}{|l|}{ Medication } \\
\hline - Anti-thrombotic agents & $10(50 \%)$ & $15(21 \%)$ & $0.0085^{1}$ \\
\hline - Non-steroidal anti-inflammatory drugs & $7(35 \%)$ & $16(22 \%)$ & 0.23 \\
\hline - Proton pump inhibitor & $5(25 \%)$ & $13(18 \%)$ & 0.47 \\
\hline Endoscopist's Experience (>3000 colonoscopies) & $10(50 \%)$ & $43(59 \%)$ & 0.48 \\
\hline
\end{tabular}

\section{Discussion}

Although several risk factors for colonic diverticular rebleeding have been identified, the endoscopic appearance of diverticulum with bleeding has rarely been evaluated. We reviewed endoscopic images of patients with colonic diverticular bleeding and classified the appearance of bleeding into two types according to the visibility of bleeding source, to find that patients with type 1 visibility (active bleeding with invisible bleeding source) were more likely to re-bleed than those with type 2 visibility (inactive bleeding with visible bleeding vessels). Our findings could be useful for risk stratification of the diverticular rebleeding and probably lead to the better choice of hemostatic methods according to the risk of rebleeding.

In the field of gastroduodenal ulcer bleeding, rebleeding risk has been evaluated according to appearance of ulcers by using the Forrest classification, and previous studies have shown that presence of active bleeding (Forrest la and lb) is a significant risk factor of rebleeding [13-15]. Meanwhile, classification like the Forrest classification does not appear applicable to colonic diverticular bleeding, because the bleeding source is sometimes located in the closed diverticular bed and cannot be observed directly.

In this regard, hemostatic procedures between gastroduodenal ulcers and colonic diverticular bleeding are somewhat different. In most cases of the former, the bleeding point can be seen and hemostasis could be directly targeted to the bleeding source. On the other hand, in the latter, direct hemostatic approach to the bleeding source is sometimes impossible due to invisibility. While presence of active bleeding is a risk factor in gastroduodenal ulcers, therefore, we assumed that invisibility of bleeding source may be a factor of rebleeding in colonic diverticular bleeding. 
- Table 3 Risk factors for rebleeding within one year for patients with colonic diverticular bleeding who underwent endoscopic hemostasis (multivariate analysis).

\begin{tabular}{|l|l|l|}
\hline & $\begin{array}{l}\text { Odds Ratio } \\
\text { (95\% Confidence interval) }\end{array}$ & $P$ \\
\hline Type 1 & $3.05(1.03-9.59)$ & $0.044^{1}$ \\
\hline Impaired renal function & $3.67(0.99-13.58)$ & 0.051 \\
\hline Antithrombotic agents & $2.94(0.95-9.12)$ & 0.061 \\
\hline 1 means statistically significant & \\
\hline
\end{tabular}

- Table 4 Risk factors for early rebleeding for patients with colonic diverticular bleeding who underwent endoscopic hemostasis (multivariate analysis).

\begin{tabular}{|l|l|l|}
\hline & $\begin{array}{l}\text { Odds Ratio } \\
\text { (95\% Confidence interval) }\end{array}$ & P \\
\hline Type 1 & $6.37(1.26-32.59)$ & $0.022^{1}$ \\
\hline Impaired renal function & $4.19(0.84-30.87)$ & 0.1 \\
\hline 1 means statistically significant & & \\
\hline
\end{tabular}

According to our results, lack of definite detection of bleeding source was likely to be a cause of diverticular rebleeding, and this result may be due to insufficient hemostatic procedures. For type 1 bleeding, hemostasis was achieved by closing the bleeding diverticulum with hemoclips. Such procedures could not sufficiently crush the vessel responsible for bleeding, and eventually could lead to easier rebleeding. In fact, it has been reported that closure of an ulcer formed after endoscopic submucosal dissection by clipping is not effective in preventing the incidence of delayed bleeding [16-18]. Thus, to obtain efficient hemostasis by hemoclips, direct application to the vessels responsible for bleeding is required.

Reported endoscopic hemostasis procedures for diverticular bleeding other than clipping include epinephrine injection, contact thermal therapy, and endoscopic band ligation $[6,19$, 20]. However, the risk of perforation is a concern for contact thermal therapy and/or epinephrine injection due to the thinness of colonic wall and the absence of a muscular layer in the colonic diverticula $[19,21]$. In this context, clipping is a relatively safe procedure that provides immediate mechanical hemostasis owing to less injury to the colonic tissues [22,23]. In this study, therefore, all patients were treated by endoscopic clipping. However, the findings of this study suggest that clipping is not always applicable to colonic diverticular bleeding.

Recently, band ligation is applied for diverticular bleeding because of the low rebleeding rate compared to endoscopic clipping [24]. Band ligation is recommended due to both lower early and late rebleeding rates and its safety profile with a lower risk of perforation [24, 25]. In consideration of the results of the current study, band ligation may be particularly applied to diverticular bleeding with type 1 visibility. On this issue, further prospective studies to compare the hemostatic performance

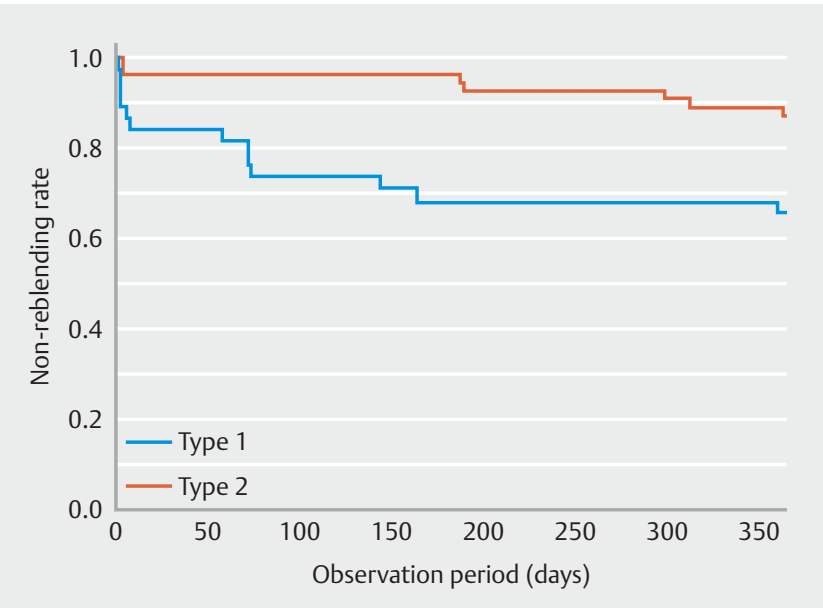

- Fig. 3 Rebleeding rate according to patient type (invisible and visible sources of bleeding). Kaplan-Meier curve showed that the cumulative incidence of rebleeding was significantly higher in patients with type 1 visibility than in patients with type 2 visibility $(P=0.0033$, log-rank test $)$

between clipping and ligation according to endoscopic visibility of bleeding are required.

In the current study, endoscopic invisibility was the only risk factor for diverticular rebleeding, although other risk factors for the diverticular rebleeding including hypertension, arteriosclerosis and regular use of anti-thrombotic agents or NSAIDs have been reported [1-3]. Because those parameters were also included in the analysis of this study, endoscopic invisibility appears to be the most relevant factor that could affect the risk of rebleeding. In this context, previous studies did not include endoscopic invisibility as the risk of rebleeding, and therefore, confirmatory studies for our results by using other cohorts are required.

There are limitations to this study. First, the number of the examined patients was relatively small and all subjects were Japanese. Reproducibility should be confirmed with larger cohorts including other ethnic populations. Another drawback, due to the study being retrospective, is the possibility of missing relevant clinical parameters. In particular, visibility of bleeding and hemostasis, and accuracy of clipping procedures were reviewed by stored endoscopic images. Misclassification, therefore, may exist due to lack of appropriate endoscopic images. Finally, a black spot we defined as type 2 appears to be the same as a flat spot indicated in a previous study, and the risk of rebleeding of the lesion was considered to be very low without any hemostatic treatments [26]. This fact might contribute to the low rebleeding rate of type 2 .

In conclusion, endoscopic invisibility of colonic diverticular bleeding is significantly associated with the risk of rebleeding. Direct observation and direct approach to responsible vessels are necessary for assured hemostasis with clipping. When bleeding source cannot be observed directly, clipping should not be applied, instead band ligation may be the first choice for hemostasis. 
Competing interests

None

\section{References}

[1] Strate LL, Liu YL, Huang ES et al. Use of Aspirin or Nonsteroidal Antiinflammatory Drugs Increases Risk for Diverticulitis and Diverticular Bleeding. Gastroenterology 2011; 140: 1427-1433

[2] Niikura R, Nagata N, Akiyama J et al. Hypertension and concomitant arteriosclerotic diseases are risk factors for colonic diverticular bleeding: a case control study. Int J Colorectal Dis 2012; 22: 1137 1143

[3] Nagata N, Niikura R, Aoki T et al. Increase in colonic diverticulosis and diverticular hemorrhage in an aging society: lessons from a 9-year colonoscopic study of 28,192 patients in Japan. Int J Colorectal Dis 2014; $29: 379-385$

[4] Newman J, Fitzgerald JEF, Gupta S et al. Outcome predictors in acute surgical admissions for lower gastrointestinal bleeding. Color Dis 2012; 14: 1020 - 1026

[5] Poncet G, Heluwaert F, Voirin D et al. Natural history of acute colonic diverticular bleeding: a prospective study in 133 consecutive patients. Aliment Pharmacol Ther 2010; 32: 466-471

[6] Jensen DM, Machicado GA, Jutabha R et al. Urgent Colonoscopy for the Diagnosis and Treatment of Severe Diverticular Hemorrhage. N Engl J Med 2000; 342: $78-82$

[7] Niikura R, Nagata N, Yamada A et al. Recurrence of colonic diverticular bleeding and associated risk factors. Color Dis 2012; 14: 302 - 305

[8] Gilshtein H, Kluger $\mathrm{Y}$, Khoury A et al. Massive and recurrent diverticular hemorrhage, risk factors and treatment. Int J Surg 2016; 33 : $136-139$

[9] Watanabe S, Kodera S, Shimura H. Investigation of risk factors predicting recurrence of colonic diverticular hemorrhage and development of a recurrence risk score. Acta Gastroenterol Belg 2014; 77: $408-412$

[10] Fujino $\mathrm{Y}$, Inoue $\mathrm{Y}$, Onodera $\mathrm{M}$ et al. Risk factors for early re-bleeding and associated hospitalization in patients with colonic diverticular bleeding. Color Dis 2013; 15: 982 - 986

[11] Strate LL, Gralnek IM. ACG Clinical Guideline: Management of Patients With Acute Lower Gastrointestinal Bleeding. Am J Gastroenterol 2016; 111: 459-474

[12] Ishii N, Hirata N, Omata F et al. Location in the ascending colon is a predictor of refractory colonic diverticular hemorrhage after endoscopic clipping. Gastrointest Endosc 2012; 76: 1175-1181
[13] Chung I, Kim E, Lee M et al. Endoscopic Factors Predisposing to Rebleeding Following Endoscopic Hemostasis in Bleeding Peptic Ulcers. Endoscopy 2001; 33: 969-975

[14] Guglielmi A, Ruzzenente A, Sandri M et al. Risk Assessment and Prediction of Rebleeding in Bleeding Gastroduodenal Ulcer. Endoscopy 2002; 34: $778-786$

[15] Elmunzer B], Young SD, Inadomi JM et al. Systematic review of the predictors of recurrent hemorrhage after endoscopic hemostatic therapy for bleeding peptic ulcers. Am J Gastroenterol 2008; 103: $2625-2632$

[16] Akimoto T, Goto O, Sasaki M et al. Hold-and-drag closure technique using repositionable clips for large mucosal defects after colonic endoscopic submucosal dissection. Endosc Int Open 2016; 4: E1068E1072

[17] Osada T, Sakamoto N, Ritsuno H et al. Closure with clips to accelerate healing of mucosal defects caused by colorectal endoscopic submucosal dissection. Surg Endosc 2016; 30: 4438 - 4444

[18] Suzuki S, Chino A, Kishihara T et al. Risk factors for bleeding after endoscopic submucosal dissection of colorectal neoplasms. World Gastroenterol 2014; 20: 1839-1845

[19] Rustagi T, McCarty TR. Endoscopic management of diverticular bleeding. Gastroenterol Res Pract 2014; 9: 353508

[20] Ishii N, Itoh T, lizuka Y et al. Endoscopic band ligation for colonic diverticular rebleeding after endoscopic clipping. Dig Endosc 2011; 23: 211

[21] Schmulewitz N, Fisher DA, Rockey DC. Early colonoscopy for acute lower $\mathrm{GI}$ bleeding predicts shorter hospital stay: a retrospective study of experience in a single center. Gastrointest Endosc 2003; 58: 841 846

[22] Kaltenbach T, Watson R, Shah J et al. Colonoscopy with clipping is useful in the diagnosis and treatment of diverticular bleeding. Clin Gastroenterol Hepatol 2012; 10: 131 - 137

[23] Shimamura Y, Ishii N, Omata F et al. Endoscopic band ligation for colonic diverticular bleeding: possibility of standardization. Endosc Int Open 2016; 15: E233-E237

[24] Shibata S, Shigeno T, Fujimori K et al. Colonic diverticular hemorrhage: the hood method for detecting responsible diverticula and endoscopic band ligation for hemostasis. Endoscopy 2013; 11: $66-69$

[25] Ishii N, Setoyama T, Deshpande GA et al. Endoscopic band ligation for colonic diverticular hemorrhage. Gastrointest Endosc 2012; 75: 382 387

[26] Jensen DM, Ohning GV, Kovacs TOG et al. Natural history of definitive diverticular hemorrhage based on stigmata of recent hemorrhage and colonoscopic Doppler blood flow monitoring for risk stratification and definitive hemostasis. Gastrointest Endosc 2016; 83: 416-423 